\title{
Elevated platelet-derived growth factor-BB concentrations in premature neonates who develop chronic lung disease Kim G Adcock ${ }^{1}$, Jeremy Martin², John Loggins², Thomas E Kruger ${ }^{2}$ and R John Baier*2
}

Address: ${ }^{1}$ University of Mississippi Medical Center, Jackson, MS, USA and ${ }^{2}$ Louisiana State University Health Sciences Center - Shreveport, Shreveport, LA, USA

Email: Kim G Adcock - kadcock@pharmacy.umsmed.edu; Jeremy Martin - jmarti9@lsuhsc.edu; John Loggins - jloggi@lsuhsc.edu; Thomas E Kruger - dkruger1@kc.rr.net; R John Baier* - jbaiermd@yahoo.com

* Corresponding author

Published: 15 June 2004

BMC Pediatrics 2004, 4:10
Received: 10 September 2003

Accepted: 15 June 2004

This article is available from: http://www.biomedcentral.com//47/-243 I/4/10

(C) 2004 Adcock et al; licensee BioMed Central Ltd. This is an Open Access article: verbatim copying and redistribution of this article are permitted in all media for any purpose, provided this notice is preserved along with the article's original URL.

\begin{abstract}
Background: Chronic lung disease (CLD) in the preterm newborn is associated with inflammation and fibrosis. Platelet-derived growth factor-BB (PDGF-BB), a potent chemotactic growth factor, may mediate the fibrotic component of CLD. The objectives of this study were to determine if tracheal aspirate (TA) concentrations of PDGF-BB increase the first 2 weeks of life in premature neonates undergoing mechanical ventilation for respiratory distress syndrome (RDS), its relationship to the development of CLD, pulmonary hemorrhage (PH) and its relationship to airway colonization with Ureaplasma urealyticum $(\mathrm{Uu})$.
\end{abstract}

Methods: Infants with a birth weight less than 1500 grams who required mechanical ventilation for RDS were enrolled into this study with parental consent. Tracheal aspirates were collected daily during clinically indicated suctioning. Uu cultures were performed on TA collected in the first week of life. TA supernatants were assayed for PDGF-BB and secretory component of IgA concentrations using ELISA techniques.

Results: Fifty premature neonates were enrolled into the study. Twenty-eight infants were oxygen dependent at 28 days of life and 16 infants were oxygen dependent at 36 weeks postconceptual age. PDGF-BB concentrations peaked between 4 and 6 days of life. Maximum PDGF-BB concentrations were significantly higher in infants who developed CLD or died from respiratory failure. PH was associated with increased risk of CLD and was associated with higher PDGF-BB concentrations. There was no correlation between maximum PDGF-BB concentrations and $\mathrm{Uu}$ isolation from the airway.

Conclusions: PDGF-BB concentrations increase in TAs of infants who undergo mechanical ventilation for RDS during the first 2 weeks of life and maximal concentrations are greater in those infants who subsequently develop CLD. Elevation in lung PDGF-BB may play a role in the development of CLD. 


\section{Background}

Chronic lung disease (CLD) in the preterm newborn, commonly referred to as bronchopulmonary dysplasia (BPD), occurs as a result of acute lung injury (ALI) due to several factors including mechanical ventilation and oxygen therapy during the first days of life [1]. It affects as many as $43 \%$ of infants born weighing less than 1000 grams and 23\% of infants weighing less than 1500 grams. The development of CLD is associated with poor neurodevelopmental outcome and chronic pulmonary morbidity [2].

Acute lung injury in the premature newborn is associated with inflammation and may result in lung fibrosis. Inflammation, characterized by an influx of neutrophils and macrophages with the release of pro-inflammatory and chemotactic cytokines, is followed by variable degrees of intraalveolar and interstitial fibrosis, airway maldevelopment and failure of septation, all of which ultimately alter the development of the immature lung [3-5]. Previous research into respiratory distress syndrome (RDS) in the newborn has identified several inflammatory mediators which may play a role in the development of CLD [3,6-11]. The potential role of growth factors in the development of CLD has been studied to a lesser degree. One of the many growth factors that may play a role in the development of CLD in the newborn is platelet derived growth factor (PDGF). PDGF a family of homo and heterodimers of disulfide-bonded $\mathrm{A}$ and $\mathrm{B}$ polypeptide chains resulting in 3 isoforms $\mathrm{AA}, \mathrm{AB}$, and $\mathrm{BB}$, is synthesized by a variety of cells including macrophages, endothelial cells and fibroblasts [12]. PDGF-BB is a potent chemotactic and a "competence" growth factor for fibroblasts and smooth muscle cells, and has been implicated in the pathogenesis of fibrotic lung conditions [12-21]. Fibrosis is also a component in CLD in some newborns. However, the role PDGF-BB may play in CLD in the preterm newborn remains undefined.

This study was conducted to determine if PDGF-BB concentrations increase during the first 2 weeks of life in mechanically ventilated premature neonates with RDS and to evaluate the relationship between PDGF-BB and the subsequent development of CLD. In addition, the impact of airway colonization with Ureaplasma urealyticum (Uu) and pulmonary hemorrhage (PH) on tracheal aspirate concentrations of PDGF-BB was assessed.

\section{Methods}

The study population consisted of very low birth weight infants admitted to the neonatal intensive care unit at the Louisiana State University Health Sciences Center in Shreveport. Many of these subjects have been reported in our earlier studies of inflammatory mediators and the development of BPD $[22,23]$. Eligibility criteria included a birth weight of less than 1500 grams, RDS requiring mechanical ventilation during the first week of life and parental consent. The study was approved by the Institutional Review Board for Human Research at Louisiana State University Health Sciences Center.

Tracheal aspirates were collected daily during clinically indicated suctioning procedures using a standardized technique. Sterile normal saline $(0.5 \mathrm{ml})$ was instilled into the endotracheal tube and 3-4 manual breaths were provided by a self inflating resuscitation bag. Airway secretions were aspirated into sterile traps and any material remaining in the catheter was washed into the trap with an additional $0.5 \mathrm{cc}$ saline. Tracheal aspirates were centrifuged $14,000 \mathrm{~g}$ for 10 minutes to remove cellular material and the supernatant frozen at $-70^{\circ} \mathrm{C}$ until cytokine assays performed. Uu cultures were performed on TA collected in the first week of life. TA supernatants were assayed for PDGF-BB concentrations using enzymelinked immunoadsorbent assay (ELISA) developed in our laboratory using commercially available reagents. TA PDGF-BB concentrations were normalized to secretory component to account for variation in collection volumes.

Clinical data collected included the following: need for surfactant therapy, duration of mechanical ventilation, use of postnatal corticosteroids, and the incidence of patent ductus arteriosus (PDA) and pulmonary hemorrhage $(\mathrm{PH})$.

\section{Ureaplasma Culture}

TA samples were cultured for the presence of Uu using the following selective growth media: SP4 with urea (Remel, Lexana, KS), Mycotrim GU ${ }^{\circledast}$ system (Irvine Scientific, Santa Anna, CA), as well as urea and arginine-enriched pleuropneumonia-like organism broths [24]. Fifty microliters of TA were cultured in $2 \mathrm{ml}$ of the appropriate broths. An additional $50 \mu \mathrm{L}$ of TA was placed into a Mycotrim flask and subsequently incubated at $37^{\circ} \mathrm{C}$ in $5 \% \mathrm{CO}_{2}$. The media was inspected twice a day for the first 2 days and then inspected daily for 5 days to observe any color change in the media indicative of a positive culture. Ureaplasma identification was confirmed by microscopic examination of the distinct colony characteristics of ureaplasmas on the solid media in the Mycotrim flask.

\section{ELISA}

Recombinant PDGF $\mathrm{r} \beta / \mathrm{Fc}$ Chimera and biotinylated monoclonal anti-human PDGF-BB were purchased from R\&D Systems (Minneapolis, MN). Recombinant human PDGF was purchased from Pepro Tech (Rocky Hill, NJ). Streptavidin-horseradish peroxidase conjugate (StreptHRP) were purchased from Pharmingen (San Diego, CA). Horseradish peroxidase-conjugated rabbit antigoat 
antibody, monoclonal and polyclonal goat antihuman secretory component antibodies, and bovine serum albumin were purchased from Sigma Chemical Co (St. Louis, $\mathrm{MO}$ ). Human secretory immunoglobulin A (IgA) was purchased from ICN Biomedicals (Costa Mesa, CA). ELISA plates were obtained from Costar (Cambridge, MA). 2,2'azino-di [3-ethyl-benzthiazoline] (TMB) peroxidase substrate kits were purchased from Bio-Rad (Hercules, CA).

Tracheal aspirate concentrations were measured by a modification of the enzyme linked immunosorbent assay which employs a PDGF receptor $\beta$ Fc chimera as the first layer of the assay. ELISA plates were coated with $100 \mu \mathrm{L}$ of recombinant PDGF $\mathrm{r} \beta / \mathrm{Fc}$ Chimera $(0.4 \mu \mathrm{g} / \mathrm{ml})$ in phospate-buffered saline ( $\mathrm{pH} 7.4)$ and incubated overnight at $4^{\circ} \mathrm{C}$. After coating, the ELISA plates were washed once (4 minutes) with $200 \mu \mathrm{L}$ of phosphate-buffered saline containing $0.05 \%$ Tween 20 (wash buffer). All subsequent washes were performed similarly. The plates were then blocked using $200 \mu \mathrm{L}$ of phosphate-buffered saline/ Tween 20 containing 3\% (w/v) bovine serum albumin (blocking buffer) for 1-2 hours at room temperature. After blocking, the plates were washed three times before use. TA samples and recombinant PDGF-BB standards were appropriately diluted in blocking buffer. One hundred microliters of diluted TA sample (diluted 1:2 in blocking buffer) or standards $(3.9-1000 \mathrm{pg} / \mathrm{ml})$ was incubated overnight at $4^{\circ} \mathrm{C}$. Plates were washed four times and incubated with $100 \mu \mathrm{L}$ of biotinylated monoclonal anti-human PDGF-BB $(100 \mathrm{ng} / \mathrm{ml})$ for 2 hours at room temperature. Plates were washed four times and then incubated with Strept-HRP for 30 minutes at room temperature. Plates were washed four times and then developed with TMB substrate. The reaction was stopped with $\mathrm{H}_{2} \mathrm{SO}_{4}(1: 40)$ and the plated read at $450 \mathrm{~nm}$ using an
ELISA autoreader (Cayman Chemical, Ann Arbor, MI). Measurement of secretory component was performed using an assay previously described [23]. According to manufacturer's specification, the PDGF-BB assay has shows less than $2 \%$ cross-reactivity with PDGF-AB and less than $0.6 \%$ cross-reactivity with PDGF-AA. Sensitivity of the PDGF-BB assay was $16 \mathrm{pg} / \mathrm{ml}$ and secretory component $3 \mathrm{ng} / \mathrm{ml}$.

\section{Data analysis}

To adjust for variation in collection of TAs, PDGF-BB concentrations were normalized to secretory component [25]. Samples with secretory component concentrations less than $20 \mu \mathrm{g} / \mathrm{ml}$ were considered too dilute and were excluded from analysis. All statistical analysis was performed using GB-Stat for Windows version 7.0 (Dynamic Microsystems, Silver Spring, MD). The Fishers exact test (2 tailed) was used to assess the statistical differences in categorical variables. The Student t-test was used to assess normally distributed variables. The Wilcox Rank Sum test was used for analysis of factors that were not normally distributed. The differences in tracheal aspirate PDGF concentrations were assessed using ANOVA. Post hoc analysis of daily TA cytokine concentrations was performed using the Newman-Keuls test. A probability value of less than 0.05 was considered statistically significant. The data are presented as mean \pm standard error of the mean (SEM). The definition of CLD in the preterm neonate or BPD has undergone revision since it was first coined and currently several definitions are commonly used [26-28]. Both oxygen dependency at 28 days with a compatible chest X-ray and oxygen dependency at 36 weeks PCA predict long term adverse outcomes [28]. Because of this, TA PDGF-BB concentrations were correlated to both of these outcomes.

Table I: Clinical Characteristics of Study Subjects

\begin{tabular}{|c|c|c|c|c|c|}
\hline & Off Oxygen at 28 days $(n=16)$ & Oxygen at 28 days $(n=28)$ & Died $(n=6)$ & P value ${ }^{\prime}$ & P value ${ }^{2}$ \\
\hline Birth Weight & $1112 \pm 47$ & $799 \pm 35$ & $751 \pm 101$ & $<0.001$ & 0.002 \\
\hline Gestation & $27.9 \pm 0.4$ & $26.1 \pm 0.3$ & $24.5 \pm 0.6$ & 0.001 & $<0.001$ \\
\hline Surfactant & $16(100)$ & $27(96)$ & $6(100)$ & 1.00 & 1.00 \\
\hline Prenatal Steroids & $14(88)$ & $19(68)$ & $3(50)$ & 0.278 & 0.100 \\
\hline Uu isolated & $4(25)$ & $14(50)$ & $2(33)$ & 0.105 & 1.000 \\
\hline PDA & $7(44)$ & $14(50)$ & $3(50)$ & 0.835 & 1.000 \\
\hline Indomethacin ${ }^{3}$ & $13(81)$ & $28(100)$ & $6(100)$ & 0.042 & 0.532 \\
\hline Duration IMV (days) & $8 \pm 1$ & $40 \pm 6$ & $15 \pm 1$ & $<0.001$ & 0.013 \\
\hline Duration $\mathrm{O}_{2}$ (days) & $19 \pm 3$ & $79 \pm 9$ & $15 \pm 1$ & $<0.001$ & 0.479 \\
\hline Postnatal Steroids & $5(3 I)$ & $25(89)$ & $6(100)$ & $<0.001$ & 0.012 \\
\hline Age Steroids Started & $11.6 \pm 2.8$ & $11.5 \pm 1.5$ & $6.8 \pm 3.0$ & 0.973 & 0.129 \\
\hline $\mathrm{PH}$ & $0(0)$ & $7(25)$ & $3(50)$ & 0.037 & 0.013 \\
\hline IVH & $2(13)$ & $12(43)$ & $3(50)$ & 0.039 & 0.100 \\
\hline
\end{tabular}

Data are presented as Mean \pm SEM or number with percentage in parenthesis ${ }^{\prime} \mathrm{O}_{2}$ at 28 days vs not on $\mathrm{O}_{2}$ at 28 days ${ }^{2}$ deaths vs not on $\mathrm{O}_{2}$ at 28 days ${ }^{3}$ Indomethacin was used prophylactically in most cases Uu Ureaplasma urealyticum PDA Patent ductus arteriosus PH Pulmonary hemorrhage IVH Intraventricular hemorrhage 
Table 2: Characteristics of surviving infants with or without Oxygen Dependancy at 36 Weeks PCA

\begin{tabular}{lccc}
\hline & Off Oxygen at 36 weeks $(\mathbf{n}=\mathbf{2 8})$ & Oxygen at 36 weeks PCA (n = I6) & P Value \\
\hline Birth Weight & $1011 \pm 43$ & $741 \pm 38$ & $<0.001$ \\
Gestation & $27.1 \pm 0.3$ & $26.1 \pm 0.4$ & 0.057 \\
Surfactant & $27(96)$ & $16(100)$ & 1.00 \\
Prenatal Steroids & $22(78)$ & $11(69)$ & 0.492 \\
Uu isolated & $13(46)$ & $5(31)$ & 0.324 \\
PDA & $13(46)$ & $8(50)$ & 0.907 \\
Indomethacin' & $25(89)$ & $16(100)$ & 0.290 \\
Duration IMV (days) & $15 \pm 2$ & $53 \pm 8$ & $<0.001$ \\
Duration O2 (days) & $30 \pm 4$ & $106 \pm 13$ & $<0.001$ \\
Postnatal Steroids & $14(50)$ & $16(100)$ & $<0.001$ \\
Age Steroids Stated & $10.0 \pm 1.2$ & $12.8 \pm 2.1$ & 0.282 \\
PH & $3(11)$ & $4(25)$ & 0.236 \\
IVH & $6(21)$ & $8(50)$ & 0.503 \\
\hline
\end{tabular}

Data are presented as Mean \pm SEM or number with percentage in parenthesis 'Indomethacin was used prophylactically in most cases Uu Ureaplasma urealyticum PH Pulmonary hemorrhage PDA Patent ductus arteriosus IVH Intraventricular hemorrhage

\section{Results}

Fifty premature neonates were enrolled into the study. Of the fifty infants, $28(56 \%)$ infants were oxygen dependent at 28 days of life and $16(32 \%)$ infants required oxygen therapy at 36 weeks postconceptual age (PCA). Patient characteristics can be found in Tables 1 and 2. Those infants who were oxygen dependent at 28 days or died from respiratory failure $(n=6)$ were born at an earlier gestational age, had lower birth weight, more likely to suffered a PH and more likely received postnatal corticosteroids. However, the isolation of Uu from the airways or the presence of a PDA was similar between the two groups. Infants who remained oxygen dependent at 36 weeks PCA were similarly more immature, tended to be of lower birth weight, and more likely received postnatal corticosteroids than those weaned from oxygen by this time.

From the 50 infants studied, 436 tracheal aspirate samples were analyzed with PDGF-BB concentrations detected in $230(53 \%)$ samples. The frequency of detectable PDGF$\mathrm{BB}$ concentrations was significantly lower in infants who did not develop BPD $(p=0.006)$ : $37 / 100(37 \%)$ in infants who recovered from RDS and were off oxygen at 28 days, $162 / 279$ (58\%) in infants who were $\mathrm{O}_{2}$ dependent at 28 days of life, 28/52 (54\%) in infants who died, $90 / 159(56 \%)$ in infants who were $\mathrm{O}_{2}$ dependent at 36 weeks PCA. Figure 1 illustrates the changes in TA concentrations of PDGF-BB during the first 14 days of life in infants with RDS requiring mechanical ventilation. Tracheal aspirate concentrations of PDGF-BB increased significantly during the course of RDS. PDGF-BB significantly increased until days 4-6 (mean peak at day 5) and then declined to baseline levels between 12 and 14 days of life ( $\mathrm{p}<0.001$, ANOVA repeated measures). We found no relationship between PDGF-BB concentrations and gestational age (data not shown).

\section{Tracheal aspirate concentrations of PDGF-BB and the development of chronic lung disease}

Tracheal aspirate concentrations of PDGF-BB correlated with the development of chronic lung disease. Figure 2 shows the TA concentrations of PDGF-BB in infants who were or were not oxygen dependent at 28 postnatal days. Concentrations of PDGF-BB were greater in those infants who were oxygen dependent at 28 days of life. Theses same trends persisted in the more severely affected infants who remained oxygen dependent at 36 weeks PCA (Figure $3)$.

Because infants varied with respect to the timing of their inflammatory processes, we examined the relationship of maximal TA PDGF-BB concentrations and the development of chronic lung disease. In infants who subsequently were oxygen dependent at 36 weeks, maximum PDGF-BB concentrations were significantly higher compared to those who weaned from oxygen by this time (Figure 4A). Similarly maximum PDGF-BB concentrations were greater in those infants with the combined outcome of oxygen dependency at 36 weeks PCA or death from respiratory failure compared to survivors who were weaned from oxygen by 36 weeks PCA (Figure 4B). Maximum PDGF-BB concentrations were higher in infants who died compared to those who survived and weaned from oxygen $(3416 \pm 879 \mathrm{pg} / \mu \mathrm{g} \mathrm{Sec}$ vs $825 \pm 251 \mathrm{pg} / \mu \mathrm{g} \mathrm{Sec} ; \mathrm{p}=$ 0.033 ) but not different between those who died and all survivors $(3416 \pm 879 \mathrm{pg} / \mu \mathrm{g}$ Sec vs. $2374 \pm 762 \mathrm{pg} / \mu \mathrm{g} \mathrm{Sec}$; $\mathrm{p}=0.622$ ) The peak PDGF-BB response occurred later in infants who required oxygen at 28 days compared to those who were weaned from oxygen by 28 days $(2.9 \pm 0.5$ days 


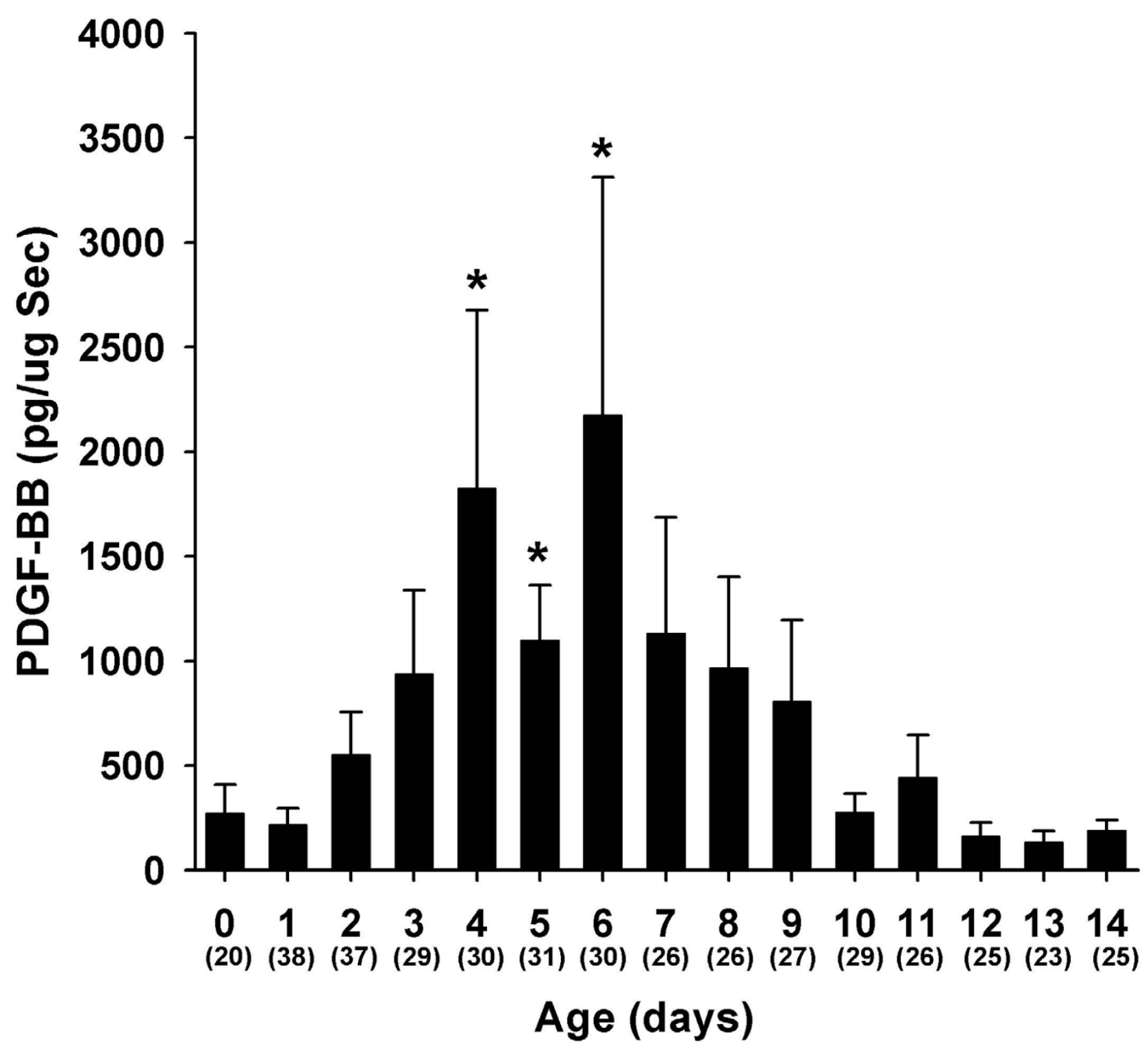

Figure I

Tracheal aspirate concentrations of PDGF-BB normalized to secretory component (sc) in mechanically ventilated infants with RDS during the first I4 days of life. Concentrations of PDGF-BB increased significantly during the course of RDS. Data represent mean \pm SEM. Numbers in parenthesis are the number of TA samples assayed per time period. *p $<0.05$ (ANOVA repeat measures).

vs. $5.6 \pm 0.6$ days; $\mathrm{p}=0.013)$. Age of peak PDGF-BB response was similar between infants who required oxygen at 36 weeks PCA and those off oxygen at 36 weeks (5.3 \pm 0.8 days vs. $4.5 \pm 0.6$ days; $p=0.436$ ) and between infants who died and those weaned from oxygen before 28 days $(5.1 \pm 0.7$ days vs. $4.5 \pm 0.6$ days; $\mathrm{p}=0.495)$

\section{Postnatal factors and TA PDGF-BB concentrations}

The role of airway colonization with Uu and PH on TA PDGF concentrations and the subsequent development of CLD were also examined. Infants with a clinically significant $\mathrm{PH}$ had a statistically significant increase in their maximum PDGF-BB concentrations (Figure 5A). Infants 


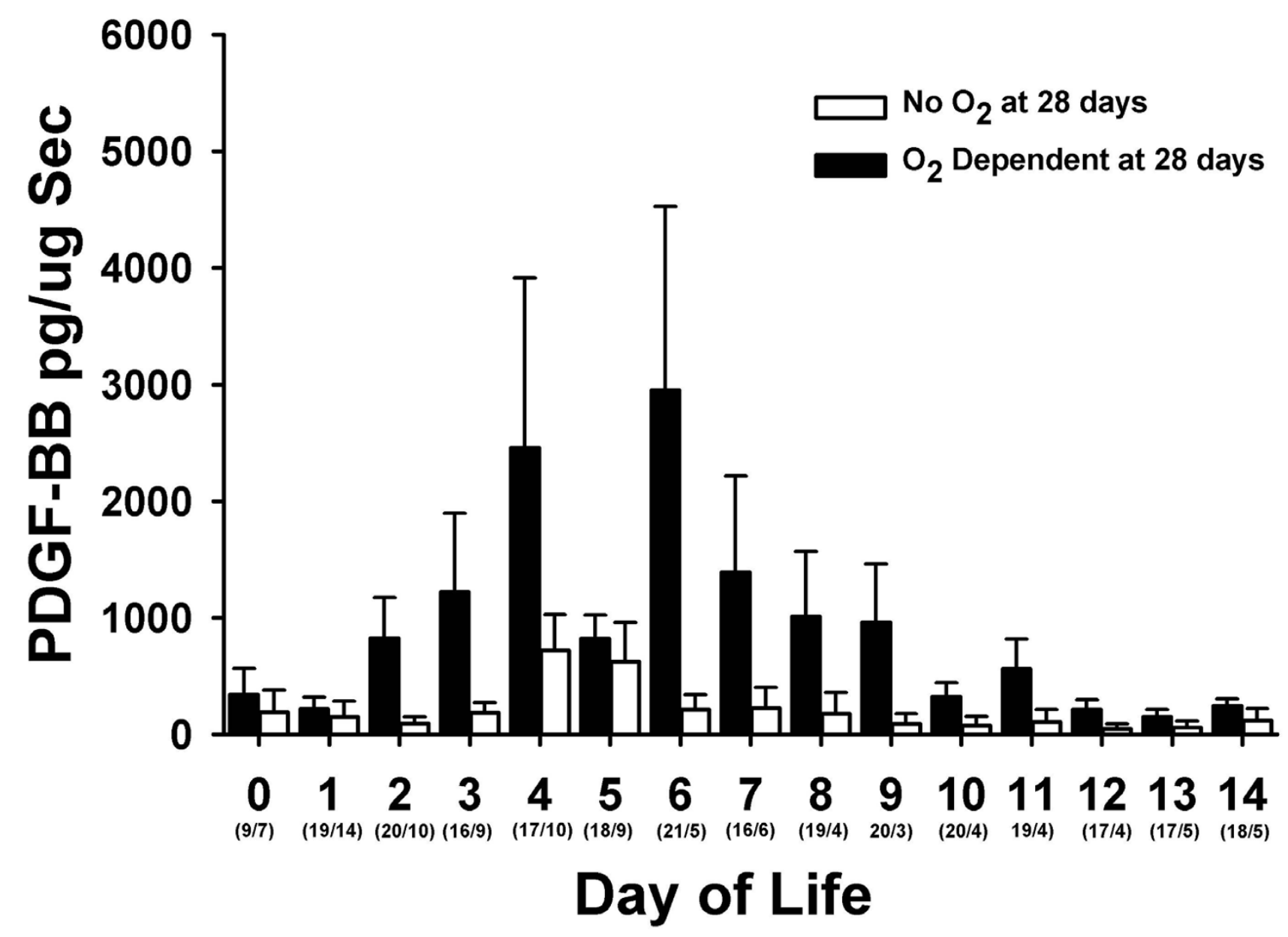

Figure 2

Tracheal aspirate concentrations of PDGF-BB normalized to secretory component (sc) during the first I4 days of life in infants who were oxygen dependent at 28 postnatal days (black bars) and those who were not (white bars). Data represent mean \pm SEM. Numbers in parenthesis are the number of TA samples assayed per time period.

who had PH were also more likely to be oxygen dependent at 28 days and at 36 weeks PCA. When infants with PH were excluded from analysis, maximum PDGF-BB concentrations remained significantly higher in infants who were oxygen dependent at 28 days $(2900 \pm 1391 \mathrm{pg} / \mu \mathrm{g}$ vs. $825 \pm 251 \mathrm{pg} / \mu \mathrm{g} ; \mathrm{p}=0.033)$. A similar non significant trend was observed for oxygen dependency at 36 weeks

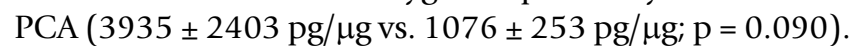

There was no correlation between maximum PDGF-BB concentrations and $\mathrm{Uu}$ isolation in this cohort of infants (Figure 5B), nor did isolation of Uu from the airways increase risk for oxygen dependency at either 28 days or 36 weeks PCA. Removal of infants with PH from the analysis did not alter this finding (data not shown).
Postnatal steroids had an inconsistent effect on those infants in whom pre and post treatment PDGF-BB concentrations were available (17 infants). Seven infants had a decline in PDGF concentrations 48-72 hours after starting steroids, 5 infants had increased PDGF-BB concentrations and 5 infants had undetectable concentrations at both sampling times.

\section{Discussion}

This study demonstrates that PDGF-BB concentrations increase during the early stages of RDS in the newborn and may therefore play a role in ALI in this setting. The timing of maximal TA PDGF-BB concentrations was similar to that observed for other inflammatory mediators 


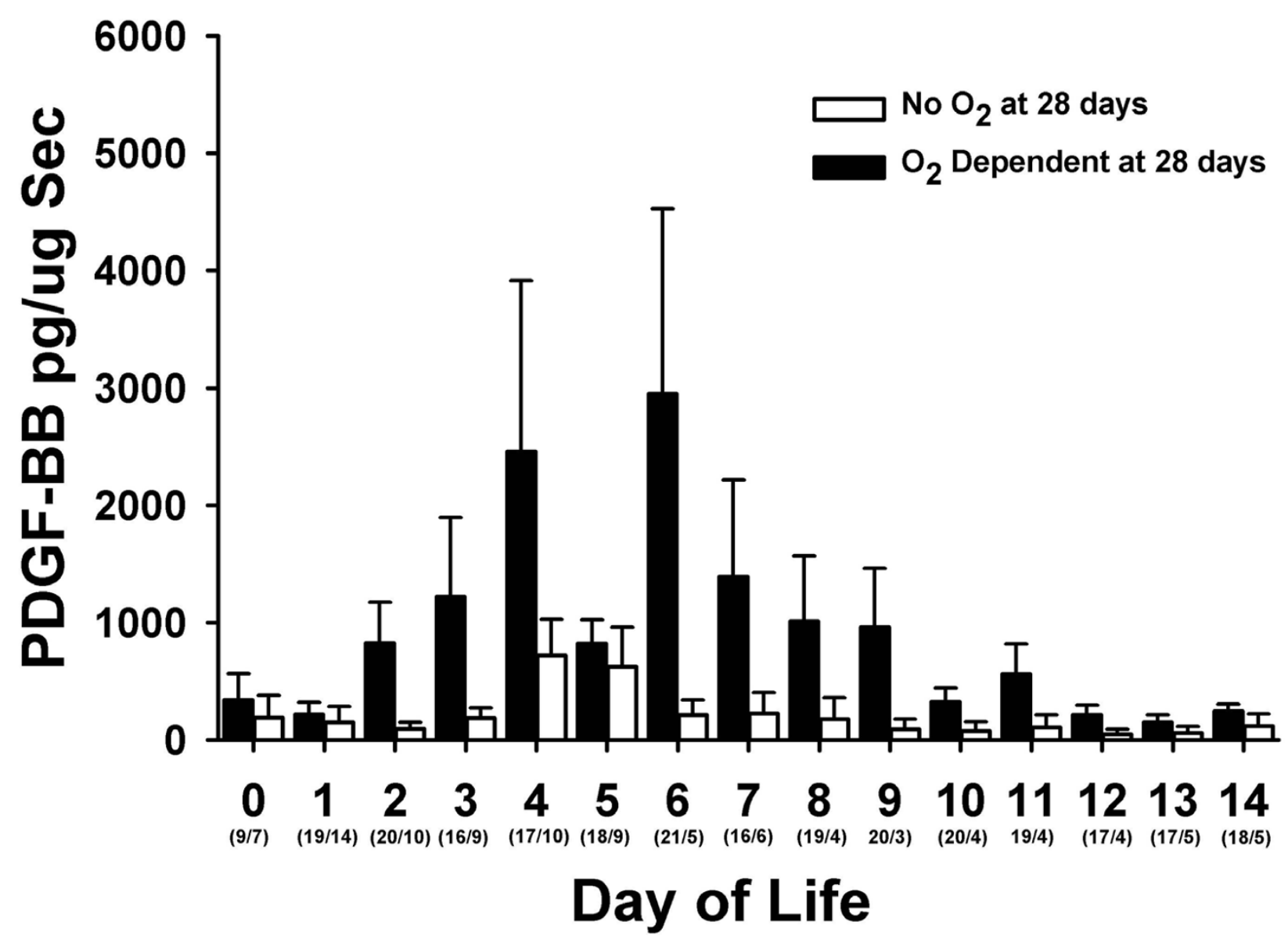

Figure 3

Tracheal aspirate concentrations of PDGF-BB normalized to secretory component (sc) during the first I4 days of life in infants who were oxygen dependent at 36 weeks PCA (black bars) and those who were not (white bars). Data represent mean \pm SEM. Numbers in parenthesis are the number of TA samples assayed per time period.

such as interleukin-8 (IL-8) and monocyte chemoattractant protein-1 (MCP-1) in earlier studies [23].

Interstitial and interalveolar fibrosis is a component of CLD in the newborn. PDGF-BB acts as a potent chemotactic and a "competence" growth factor as well as a mitogen for fibroblasts and smooth muscle cells [12]. A role of PDGF-BB in the pathogenesis of lung fibrosis has been suggested in other fibrotic lung conditions. Conditions such as idiopathetic pulmonary fibrosis, bronchiolitis obliterans, and coal-workers pneumoconiosis are associated with overexpression of PDGF [12-21].

The role of PDGF and other fibroblast growth factors in RDS and its evolution to CLD has not been extensively studied in preterm newborn. Currie et al demonstrated increased fibroblast mitogenic activity in bronchoalveolar lavage fluid (BALF) in infants with RDS and those who developed CLD compared with control infants [29]. However, there was no difference in mitogenic activity between RDS and CLD infants. In that study, the fibroproliferative activity of BALF was not blocked by anti-PDGF-BB antibody leading the authors to conclude that PDGF-BB did not significantly contribute to the mitogenic activity. However, PDGF-BB concentrations were not directly measured in that study. More recently, Dik et al measured both PDGF-BB concentrations and fibroblast proliferation in BALF obtained from 15 infants before and after systemic dexamethasone [30]. BALF PDGF-BB concentrations were measurable prior to dexamethasone 

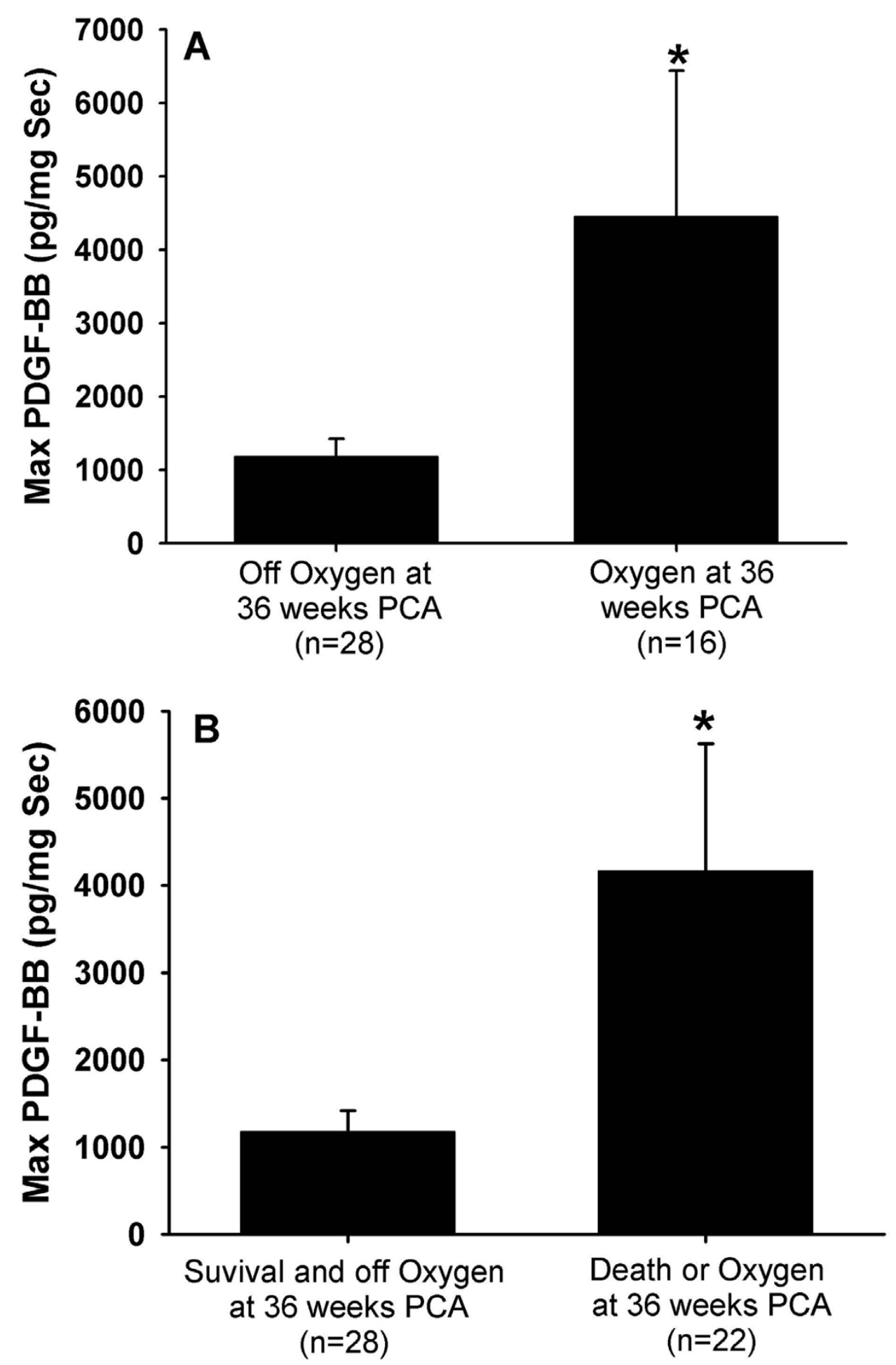

Figure 4

Maximal TA concentrations PDGF-BB normalized to secretory component (sc) comparing A) infants who were oxygen dependent at 36 weeks PCA vs those who were not and B) infants who died or were oxygen dependent at 36 weeks PCA vs survivors weaned from oxygen at 36 weeks PCA. Data represent mean \pm SEM. $*_{p}<0.05$ 

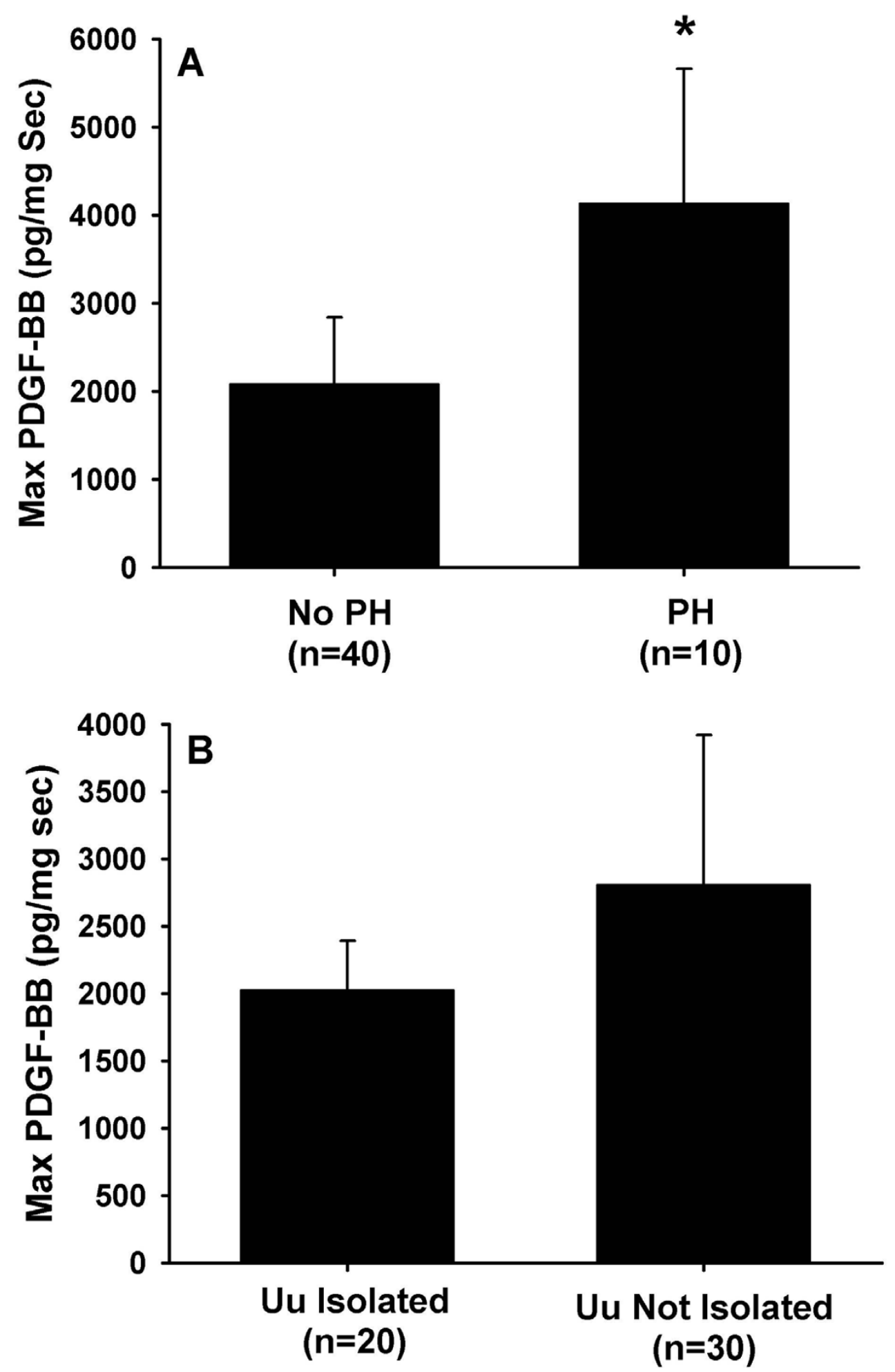

Figure 5

Maximal TA concentrations PDGF-BB normalized to secretory component (sc) comparing A) infants with and without pulmonary hemorrhage and $B$ ) infants with and without isolation of Ureaplasma urealyticum. Data represent mean \pm SEM. $*_{p}<0.05$ 
therapy. Furthermore, the mitogenic activity of BALF was inhibited by a specific inhibitor of the PDGF receptor tyrosine kinase. The present study expands and enhances these observations by providing longitudinal data of TA PDGF-BB concentrations and correlation with the development of CLD. PDGF-BB concentrations from TA fluid in infants who develop CLD were elevated when compared to those infants who recovered from their initial lung injury. These results are consistent with a role for PDGF-BB in the pathogenesis of CLD in the premature newborn.

The fibrotic process may be activated soon after birth since the peak concentrations we observed occurred in the first week of life. This is consistent with other studies that demonstrated elevated concentrations of another pro-fibrotic factor, transforming growth factor $\beta_{1}$ during the first week of life [3].

We did not observe an effect of dexamethasone on PDGF$\mathrm{BB}$ concentrations. This lack of inhibition by postnatal steroid administration on PDGF-BB concentrations was also observed in Dik et al [30]. In Dik's study, dexamethasone increased both PDGF-BB and fibroblast proliferative properties of BALF.

The early increase in PDGF-BB concentrations may imply a perinatal insult that influenced the development of inflammation and fibrosis. We have demonstrated that airway colonization or infection with Ureaplasma urealyticum is associated with increased concentrations of IL- 8 and MCP-1 and may be a contributing factor to the development of CLD [23]. However, in this study Uu isolation was not higher in infants who develop CLD. Comparison of maximum PDGF-BB concentrations and $\mathrm{Uu}$ isolation revealed no differences.

Pulmonary hemorrhage was associated with an increased risk of CLD and was associated with increased TA concentrations of PDGF-BB. This is similar to our earlier report of increased MCP-1 and IL- 8 concentrations in infants with $\mathrm{PH}$ [22]. Hemorrhage into the lung, which may not be clinically apparent, may be an important source of PDGF-BB. Platelets are a rich source of PDGF, particularly PDGF-AB and may account for some of the PDGF-BB measured in our study [31]. However, PDGF-BB concentrations were still greater in CLD infants after infants with clinically significant $\mathrm{PH}$ were excluded from analysis suggesting that $\mathrm{PH}$ was not the only determinant of TA PDGF-BB concentration. Other sources, such as respiratory epithelial cells and alveolar macrophages may also contribute to the PDGF-BB measured [14,32].

All studies of this nature suffer from limitations inherent in their design. Tracheal aspirate growth factor concentra- tions may not reflect what is happening at the alveolus or interstitium of the lung. Moreover, because in clinical practice infants are extubated as soon as feasible to limit potential damage from continuing ventilation, infants who have less severe lung disease or recover from their acute lung injury rapidly are not sampled. Similar ethical considerations preclude the use of control infants. Thus, there are disproportionately fewer samples and fewer time points in infants who recover, or do not develop chronic lung disease which introduces bias into our results limiting the conclusions that may be drawn. However, we believe our results are consistent with other similar clinical studies and in animal models of lung injury providing further insight into the mechanisms of chronic lung injury in the newborn.

In our study we employed a higher centrifugation speed than other studies. This may cause lysis of the cellular components (ie macrophages) of the TA. Thus TA PDGFBB concentrations may reflect total PDGF-BB and not just free secreted growth factor.

\section{Conclusions}

In conclusion, the fibrotic component of chronic lung disease in preterm infants may be mediated by growth factors such as PDGF. This study demonstrated that PDGF-BB concentrations increase in TAs of infants who undergo mechanical ventilation for RDS during the first week of life.

Presented in part the 2001 Southern Society of Pediatric Research Meeting New Orleans, LA

\section{Abbreviations}

Platelet Derived Growth Factor-BB PDGF-BB

Chronic lung disease CLD

Pulmonary Hemorrhage PH

Ureaplasma urealyticum $\mathrm{Uu}$

Tracheal Aspirate TA

Postconceptual age PCA

Secretory component sc

Respiratory Distress Syndrome RDS

\section{Competing interests}

None declared. 


\section{Authors' Contributions}

KA was responsible for primary preparation of the manuscript and performance of laboratory procedures. RJB was responsible for the design of the study, statistical analysis, and final editing of the manuscript. JL supervised collection of TA samples, helped with obtaining informed consent and editing of manuscript. JM assisted with performance of PDGF-BB assay. TK assisted with laboratory analysis and editing of manuscript.

\section{References}

I. Northway W. H., Jr., Rosan RC, Porter DY: Pulmonary disease following respirator therapy of hyaline-membrane disease. Bronchopulmonary dysplasia. N Engl J Med 1967, 276:357-368.

2. Lemons JA, Bauer CR, Oh W, Korones SB, Papile LA, Stoll B], Verter J, Temprosa M, Wright LL, Ehrenkranz RA, Fanaroff AA, Stark A, Carlo W, Tyson JE, Donovan EF, Shankaran S, Stevenson DK: Very low birth weight outcomes of the National Institute of Child Health and Human Development Neonatal Research Network, January 1995 through December 1996. NICHD Neonatal Research Network. Pediatrics 200I, 107:EI.

3. Kotecha S: Cytokines in chronic lung disease of prematurity. Eur J Pediatr 1996, 155 Suppl 2:SI4-7.

4. Gregoire MC, Lefebvre F, Glorieux J: Health and developmental outcomes at 18 months in very preterm infants with bronchopulmonary dysplasia. Pediatrics 1998, 101:856-860.

5. Zimmerman J]: Bronchoalveolar inflammatory pathophysiology of bronchopulmonary dysplasia. Clin Perinatol 1995, 22:429-456

6. Kotecha S, Chan B, Azam N, Silverman M, Shaw RJ: Increase in interleukin-8 and soluble intercellular adhesion molecule- $I$ in bronchoalveolar lavage fluid from premature infants who develop chronic lung disease. Arch Dis Child Fetal Neonatal Ed 1995, 72:F90-6.

7. Groneck P, Gotze-Speer B, Oppermann M, Eiffert H, Speer CP: Association of pulmonary inflammation and increased microvascular permeability during the development of bronchopulmonary dysplasia: a sequential analysis of inflammatory mediators in respiratory fluids of high-risk preterm neonates. Pediatrics 1994, 93:7|2-7|8.

8. Kotecha $S$, Wilson $L$, Wangoo A, Silverman $M$, Shaw RJ: Increase in interleukin (IL)-I beta and IL-6 in bronchoalveolar lavage fluid obtained from infants with chronic lung disease of prematurity. Pediatr Res 1996, 40:250-256.

9. Suter PM, Suter S, Girardin E, Roux-Lombard P, Grau GE, Dayer JM: High bronchoalveolar levels of tumor necrosis factor and its inhibitors, interleukin- I, interferon, and elastase, in patients with adult respiratory distress syndrome after trauma, shock, or sepsis. Am Rev Respir Dis 1992, 145:1016-1022.

10. Carre PC, Mortenson RL, King T. E., Jr., Noble PW, Sable CL, Riches DW: Increased expression of the interleukin-8 gene by alveolar macrophages in idiopathic pulmonary fibrosis. A potential mechanism for the recruitment and activation of neutrophils in lung fibrosis. / Clin Invest |99|, 88: |802-I8I0.

II. Donnelly SC, Strieter RM, Kunkel SL, Walz A, Robertson CR, Carter DC, Grant IS, Pollok AJ, Haslett C: Interleukin-8 and development of adult respiratory distress syndrome in at-risk patient groups. Lancet 1993, 341:643-647.

12. Heldin $\mathrm{CH}$, Westermark B: Mechanism of action and in vivo role of platelet-derived growth factor. Physiol Rev 1999, 79: $1283-1316$.

13. Vaillant P, Menard O, Vignaud JM, Martinet N, Martinet $Y$ : The role of cytokines in human lung fibrosis. Monaldi Arch Chest Dis 1996, 5 I: $145-152$.

14. Nagaoka I, Trapnell BC, Crystal RG: Upregulation of plateletderived growth factor-A and $-B$ gene expression in alveolar macrophages of individuals with idiopathic pulmonary fibrosis. J Clin Invest 1990, 85:2023-2027.

15. Vignaud JM, Allam M, Martinet N, Pech M, Plenat F, Martinet Y: Presence of platelet-derived growth factor in normal and fibrotic lung is specifically associated with interstitial macrophages, while both interstitial macrophages and alveolar epithelial cells express the c-sis proto-oncogene. Am J Respir Cell Mol Biol 1991, 5:53I-538.

16. Antoniades HN, Bravo MA, Avila RE, Galanopoulos T, Neville-Golden J, Maxwell M, Selman M: Platelet-derived growth factor in idiopathic pulmonary fibrosis. J Clin Invest 1990, 86: I055- 1064.

17. Aubert JD, Pare PD, Hogg JC, Hayashi S: Platelet-derived growth factor in bronchiolitis obliterans-organizing pneumonia. $\mathrm{Am} J$ Respir Crit Care Med 1997, I 55:676-68I.

18. Hertz MI, Henke CA, Nakhleh RE, Harmon KR, Marinelli WA, Fox JM, Kubo SH, Shumway SJ, Bolman R. M., 3rd, Bitterman PB: Obliterative bronchiolitis after lung transplantation: a fibroproliferative disorder associated with platelet-derived growth factor. Proc Natl Acad Sci U S A 1992, 89: $10385-10389$.

19. Vanhee D, Gosset P, Wallaert B, Voisin C, Tonnel AB: Mechanisms of fibrosis in coal workers' pneumoconiosis. Increased production of platelet-derived growth factor, insulin-like growth factor type I, and transforming growth factor beta and relationship to disease severity. Am J Respir Crit Care Med 1994, I50:1049-1055.

20. Snyder LS, Hertz MI, Peterson MS, Harmon KR, Marinelli WA, Henke CA, Greenheck JR, Chen B, Bitterman PB: Acute lung injury. Pathogenesis of intraalveolar fibrosis. J Clin Invest 1991, 88:663-673.

21. Walsh J, Absher M, Kelley J: Variable expression of plateletderived growth factor family proteins in acute lung injury. Am J Respir Cell Mol Biol 1993, 9:637-644.

22. Baier RJ, Loggins J, Kruger TE: Increased interleukin-8 and monocyte chemoattractant protein-I concentrations in mechanically ventilated preterm infants with pulmonary hemorrhage. Pediatr Pulmonol 2002, 34: I3I-I37.

23. Baier RJ, Loggins J, Kruger TE: Monocyte chemoattractant protein- $I$ and interleukin-8 are increased in bronchopulmonary dysplasia: relation to isolation of Ureaplasma urealyticum. $J$ Investig Med 200I, 49:362-369.

24. Isenberg Henry D., American Society for Microbiology.: Clinical microbiology procedures handbook. Washington, D.C., American Society of Microbiology; 1992:2 v. (various pagings).

25. Watts CL, Bruce MC: Comparison of secretory component for immunoglobulin $A$ with albumin as reference proteins in tracheal aspirate from preterm infants. J Pediatr 1995, 127:113-122.

26. Bancalari E, Abdenour GE, Feller R, Gannon J: Bronchopulmonary dysplasia: clinical presentation. J Pediatr 1979, 95:819-823.

27. Shennan AT, Dunn MS, Ohlsson A, Lennox K, Hoskins EM: Abnormal pulmonary outcomes in premature infants: prediction from oxygen requirement in the neonatal period. Pediatrics 1988, 82:527-532.

28. Palta M, Sadek M, Barnet JH, Evans M, Weinstein MR, McGuinness G, Peters ME, Gabbert D, Fryback D, Farrell P: Evaluation of criteria for chronic lung disease in surviving very low birth weight infants. Newborn Lung Project. J Pediatr 1998, 132:57-63.

29. Currie AE, Kelly M, Vyas JR, Pandya H, Field D, Kotecha S: Fibroblast mitogenic activity of lung lavage fluid from infants with chronic lung disease of prematurity. Arch Dis Child Fetal Neonatal Ed 2002, 86:FI93-7.

30. Dik WA, Versnel MA, Naber BA, Janssen DJ, van Kaam AH, Zimmermann LJ: Dexamethasone treatment does not inhibit fibroproliferation in chronic lung disease of prematurity. Eur Respir 2003, 21:842-847.

3I. Hart CE, Bailey M, Curtis DA, Osborn S, Raines E, Ross R, Forstrom JW: Purification of PDGF-AB and PDGF-BB from human platelet extracts and identification of all three PDGF dimers in human platelets. Biochemistry 1990, 29:166-172.

32. Buch S, Han RN, Cabacungan J, Wang J, Yuan S, Belcastro R, Deimling J, Jankov R, Luo X, Lye SJ, Post M, Tanswell AK: Changes in expression of platelet-derived growth factor and its receptors in the lungs of newborn rats exposed to air or $60 \%$ O(2). Pediatr Res 2000, 48:423-433.

\section{Pre-publication history}

The pre-publication history for this paper can be accessed here:

http://www.biomedcentral.com/1471-2431/4/10/prepub 